\title{
Arabidopsis F-box gene FOA1 involved in ABA signaling
}

\author{
PENG Juan, YU DaShi, WANG LiQun, XIE MinMin, YUAN CongYing, WANG Yu, TANG Dong Ying, \\ ZHAO XiaoYing ${ }^{*} \&$ LIU XuanMing
}

College of Life Sciences, State Key Laboratory of Chemo/Biosensing and Chemometrics, Hunan University, Changsha 410082, China

\begin{abstract}
The expression of FOA1 (F-box overexpressed/oppressed ABA signaling) in different organs of Arabidopsis, and in response to $\mathrm{ABA}$ and $\mathrm{NaCl}$, was analyzed. The expression level of FOAl is higher in the root and is lower in the stem, and is induced rapidly by $\mathrm{ABA}$ and $\mathrm{NaCl}$. The phenotypes of T-DNA insertion mutant foal and FOAl overexpression lines FOAloxl and FOA1ox2 were analyzed. The foal mutant exhibited a lower germination rate, shorter root length, more stomatal opening, increased proline accumulation and hypersensitivity to ABA compared with the wild type. In contrast, the overexpression lines showed lower sensitivity to ABA than the wild type. The expression levels of several ABA and stress-responsive transcription factors and genes were altered in the foal mutant in response to ABA. Compared with the wild type, the expression levels of ABA-responsive transcription factors were higher, but ABA and stress-responsive genes were lower in foal mutant. This study demonstrates that $F O A l$ is an ABA signaling-related gene, and may play a negative role in ABA signaling.
\end{abstract}

F-box gene, FOA1, ABA, signaling, Arabidopsis

Citation: $\quad$ Peng J, Yu D S, Wang L Q, et al. Arabidopsis F-box gene FOA1 involved in ABA signaling. Sci China Life Sci, 2012, 55: 497-506, doi: 10.1007/ s11427-012-4332-9

Protein degradation is an important post-transcriptional regulation process that permits organisms to quickly respond to a changing environment, either inside or outside the cell, by adjusting the quantity of key proteins. Most cellular proteins are degraded via the UPS (ubiquitin proteasome system) pathway [1,2]. Three types of enzymes, E1 (ubiquitin activating enzymes), E2 (ubiquitin conjugating enzymes) and E3 (ubiquitin ligases), are involved in the UPS pathway for ubiquitination and degradation of target proteins. E3s display substrate specificity, specifically recognizing target proteins and subsequently mediating their proteolysis. E3s have become a current research hot spot. There are about 1200 E3s in Arabidopsis thaliana [2-4], which can be classified into several large families. SCF (SKP1-CUL1-F-box) is one of the largest, best understood, most-characterized, and widely studied E3s [5,6].

*Corresponding author(email: zxy_mm@163.com; xmL05@hnu.edu.cn)
As members of the SCF complex, F-box proteins are widely distributed among living organisms. They regulate many physiological phenomena, including hormone signal transduction (for example SLY1 and GID2 in Gibberellic acid (GA) signaling [7], EDL3 in abscisic acid (ABA) signaling [8], EBF1 and EBF2 in ethylene signaling [9], and TIR1 in auxin signaling [10]), self-incompatibility, floral development and circadian clock $[11,12]$ through their specific recognition of particular substrates. In addition, F-box proteins are involved in plant abiotic stress responses [13-15], including to drought [16-19], cold [20] and high salt [18] stress. Studies of the function of F-box proteins and their related target proteins will aid in the in-depth understanding of plant growth and related signal transduction processes.

There are approximately 700 F-box proteins in Arabidopsis [1], but the functions of most of them remain unknown. Arabidopsis gene AT3G17320 encoding an F-box protein, named for FOAl (F-box overexpressed/oppressed 
ABA signaling), is located on the third chromosome and has a length of $1230 \mathrm{bp}$ and has no introns. The protein contains a typical F-box structure domain [1,21] and a conserved FBA_1 domain, which may act as a new protein-protein interaction motif [21].

A 1204 bp genomic DNA sequence of AT3G17320 $(-1201$ to +3$)$ was analyzed using PLACE software (http://www.dna.affrc.go.jp/PLACE/), and certain plant hormone and stress-response elements were found, including AuxRE (Auxin responsive element), GARE (Gibberellin responsive element), ABRE (ABA responsive element), and LTRE (low temperature responsive element). Thus, we speculated that the expression of FOAl gene is regulated by hormones and environmental stresses, and that it might play an important role in hormone and stress-response pathways. To reveal the biological function of FOA1, we analyzed the expression level of FOAl in different organs of Arabidopsis and in response to $\mathrm{ABA}$ and $\mathrm{NaCl}$. Subsequently, T-DNA insertion mutant and over-expression lines of FOAl were used to investigate its role in ABA signaling.

\section{Materials and methods}

\subsection{FOA1 gene clone and plant transformation}

Fragments of the FOAl gene with attB sites were amplified from wild-type Arabidopsis (Col-0) cDNA by polymerase chain reaction (PCR) using prime STAR high fidelity polymerase (TaKaRa, Japan) and primers FOAIF and FOAIR (Table 1; attB sites are underlined). The fragments were subcloned into Entry Vector pDONR/ZEO using BP Clonase II (Invitrogen, USA) and sequenced. Positive fragments were cloned into destination vector pEarleyGate 203 (N-Myc) using LR Clonase II (Invitrogen, USA), and then introduced into Agrobacterium tumefaciens GV3101 by electroporation and transformed into the Arabidopsis thaliana mutant sgs (suppressor of gene silencing) (kindly provided by Professor Lin ChenTao's laboratory from University of California, Los Angeles, USA) using the floral dip method [22]. The sgs mutant, developed using ethyl methanesulfonate, carries a recessive monogenic mutation that acts in trans to specifically impede transgene-induced post-transcriptional gene silencing [23]. Thus, it can be used to express transgenes, avoiding the co-suppression phenomenon. The transgenic plants were screened using the herbicide Basta to identify homozygous lines. Leaves from the homozygous lines were collected, frozen in liquid nitrogen, and stored at $-80^{\circ} \mathrm{C}$ for RNA isolation and gene expression analysis.

\subsection{Identification of T-DNA insertion mutants}

T-DNA insertion mutant CS857416 was purchased from the biological resources center (ABRC) and is in the Columbia background (Col-0). Homozygous lines were identified by PCR using primers $\mathrm{F}, \mathrm{R}$, and the left boundary sequence of T-DNA, named R0, provided by ATIDB (the Arabidopsis thaliana Integrated Database) (Table 1). DNA was extracted from the leaves of T-DNA insertion mutants using the CTAB method and used as templates for PCR. Thereafter, the leaves of identified homozygous lines were collected, frozen in liquid nitrogen, and stored at $-80^{\circ} \mathrm{C}$ for RNA isolation and gene expression analysis.

\subsection{ABA and NaCl treatment, seed germination and root elongation assay}

Seeds were surface-sterilized in $75 \%$ ethanol for $30 \mathrm{~s}$, followed by $10 \% \mathrm{NaClO}$ for $10 \mathrm{~min}$, washed with sterile distilled water four to six times, and then stored in the dark at $4^{\circ} \mathrm{C}$ for $4 \mathrm{~d}$. These seeds were used for the $\mathrm{ABA}$ and $\mathrm{NaCl}$ treatment, seed germination and root elongation assay.

ABA treatment was as follows: Seeds were suspended in $0.1 \%$ agarose solution, placed on MS solid medium $(0.8 \%$ agarose), and then grown in a growth chamber at $22^{\circ} \mathrm{C}$ with $40 \%$ humidity and continuous white light $\left(50 \mu \mathrm{mol} \mathrm{s}{ }^{-1} \mathrm{~m}^{-2}\right)$ for $14 \mathrm{~d}$. To analyze the expression of FOAl in response to $\mathrm{ABA}$ and $\mathrm{NaCl}, 14$-day-old wild-type Col-0 seedlings were treated with $\mathrm{ABA}$ or $\mathrm{NaCl}$ by immersing their roots in liquid MS medium containing ABA $\left(10 \mu \mathrm{mol} \mathrm{L} \mathrm{L}^{-1}\right)$ or $\mathrm{NaCl}$ $\left(100 \mathrm{mmol} \mathrm{L}^{-1}\right)$ for $0,1,2,4,6$ and $8 \mathrm{~h}$, respectively. To analyze the expression of ABA and stress-response genes in the wild-type Col-0 and foal mutant in response to ABA, the 14-day-old seedlings were placed in liquid MS medium with ABA $\left(100 \mu \mathrm{mol} \mathrm{L}{ }^{-1}\right)$ for 0 or $6 \mathrm{~h}$. Samples were collected, frozen in liquid nitrogen, and stored at $-80^{\circ} \mathrm{C}$ for RNA isolation and gene expression analysis.

Seed germination analysis was as follows: Seeds were suspended in $0.1 \%$ agarose solution, placed on MS solid medium $(0.8 \%$ agarose) supplemented with different concentrations of $\mathrm{ABA}\left(0,0.3,0.6 \mu \mathrm{mol} \mathrm{L}^{-1}\right)$, and then grown in a temperature controlled chamber for $5 \mathrm{~d}$, after which the germination rate was scored. Each value was obtained from three independent experiments using approximately 100 seeds each.

Root elongation assay was as follows: Seeds were suspended in $0.1 \%$ agarose, placed on MS solid medium for $3 \mathrm{~d}$, then transferred to MS solid culture medium $(0.8 \%$ agarose) with different concentrations of $\mathrm{ABA}(0,5,10$

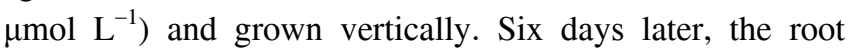
length was measured. Each value was obtained from three independent experiments with 20 samples each.

\subsection{Stomatal aperture measurement}

Stomatal apertures were measured according to the procedure described by Pei [24]. Plant rosette leaves of a 4 to 5-week-old wild-type, T-DNA insertion mutants and 
over-expression lines during the same growth stage were collected, and dipped into stomatal-opening solution (10 mmol L ${ }^{-1} \mathrm{KCl}, 7.5 \mathrm{mmol} \mathrm{L}^{-1}$ iminodiacetic acid, $10 \mathrm{mmol}$ $\mathrm{L}^{-1} \mathrm{MES}$, and $10 \mathrm{mmol} \mathrm{L}^{-1}$ Tris- $\mathrm{HCl}, \mathrm{pH}$ 6.2) for $10 \mathrm{~h}$ to induce a fully stomatal opening. They were then immersed for another $2 \mathrm{~h}$ in the same solution with different concentrations of $\mathrm{ABA}\left(0,1,10 \mu \mathrm{mol} \mathrm{L} \mathrm{L}^{-1}\right)$. Stomatal apertures were photographed and measured under a microscope (NICON TE2000). Each experiment was repeated three times.

\subsection{Assessment of the water-loss rate}

The water-loss rate was measured as described by Shan [25]. Four fully expanded rosette leaves were collected from an approximately 3-week-old wild-type, T-DNA insertion mutants and over-expression transgenic plants. After positioning them onto clean filter paper, the detached leaves were placed into a temperature controlled growth chamber at $25^{\circ} \mathrm{C}$ with $60 \%$ humidity. Fresh weight was recorded at $30 \mathrm{~min}$ intervals and the water-loss rate was calculated using the fresh-weight loss of the detached leaves. Each experiment was repeated three times.

\subsection{Proline content measurement}

Wild-type Arabidopsis, T-DNA insertion mutants and over-expression lines were treated with $\mathrm{ABA}$ for $3 \mathrm{~d}$ by spraying 0 or $100 \mu \mathrm{mol} \mathrm{L}{ }^{-1}$ ABA solution on the leaves once a day. Rosette leaves were collected and proline was extracted using the sulfosalicylic acid method [26]. The proline content was measured using the absorbance at 520 $\mathrm{nm}$. Each experiment was repeated three times.

\subsection{Total RNA isolation and RT-PCR analysis}

Total RNA was isolated using an RNA easy Mini Kit (Ambiogen Biological Technology) according to the manufacturer's instructions. First strand cDNA was synthesized using the Maxima ${ }^{\circledR}$ First cDNA Synthesis Kit (Fermentas) according to the manufacturer's specification. The cDNA product was diluted for 10 -fold, and $1 \mu \mathrm{L}$ of diluted cDNA was used in a $20 \mu \mathrm{L}$ PCR reaction. The DNA sequences of the RT-PCR primers used in this study are listed in Table 2.

Table 1 Primer sequences used for cloning the FOAl coding sequence and identifying homozygous lines of T-DNA insertion mutants

\begin{tabular}{cl}
\hline Primer name & \multicolumn{1}{c}{ Primer sequence 5'-3' $^{\prime}$} \\
\hline \multirow{2}{*}{ FOA1 } & F: CAAAAAAGCAGGCTTCATGACGAAAATTTC \\
& CGATCTT \\
& R: CAAGAAAGCTGGGTCCTAGATTTGCACAGA \\
R0 & ACTTGGA \\
ATTTGCCGATTTCGGAAC \\
& F: CAGAAACTCTTCCTCGTGGTG \\
& R: AAGAAAAAGCCGAAGCAAAAG
\end{tabular}

Table 2 Primer sequences used for RT-PCR

\begin{tabular}{cl}
\hline Primer name & \multicolumn{1}{c}{ Primer sequence 5'-3' } \\
\hline FOA1 & F: GCCTTGTGTTGATGATACCATA \\
& R: GATTTGCACAGAACTTGGAACAT \\
ACTIN2 & F: CACTGTGCCAATCTACGAGGGT \\
& R: CACAAACGAGGGCTGGAACAAG \\
\hline
\end{tabular}

PCR was generally performed with a 5 min denaturation at $95^{\circ} \mathrm{C}$, followed by 26 or 32 cycles consisting of the following steps: $95^{\circ} \mathrm{C}$ for $30 \mathrm{~s}, 58^{\circ} \mathrm{C}$ for $30 \mathrm{~s}, 72^{\circ} \mathrm{C}$ for $30 \mathrm{~s}$ for the ACTIN2 and FOA1 genes. The PCR products were separated on $1.5 \%$ agarose gels. The ACTIN2 gene was used as the internal control. RT-PCR reactions for each experiment were repeated at least three times.

\subsection{Real-time fluorescence quantitative PCR}

The cDNA product was diluted 20 -fold, and $2 \mu \mathrm{L}$ of diluted cDNA was used in a $20-\mu \mathrm{L}$ PCR reaction. The PCR was performed using a SYBR ${ }^{\circledR}$ Green I kit (TOYOBO, Japan) in an Mx3000P PCR machine (Stratagene, USA). The reaction started with a denaturation stage at $95^{\circ} \mathrm{C}$ for $10 \mathrm{~min}$, which was then followed by 40 cycles, each cycle composed of $95^{\circ} \mathrm{C}$ for $30 \mathrm{~s}, 55^{\circ} \mathrm{C}$ for $30 \mathrm{~s}, 72^{\circ} \mathrm{C}$ for $30 \mathrm{~s}$. The experiment was repeated three times from three independent experiments. The ACTIN2 gene was used as an internal control. The relative expression level was analyzed by the Mx3000P software. The sequences of the real-time fluorescence quantitative PCR primers used in this study are listed in Table 3.

\section{Results}

\subsection{Expression of $\mathrm{FOAl}$ in different organs of Ara- bidopsis}

The expression patterns of a gene in specific organs usually

Table 3 Primer sequences used for real-time fluorescence quantitative PCR

\begin{tabular}{|c|c|}
\hline Primer name & Primer sequence $5^{\prime}-3^{\prime}$ \\
\hline \multirow[t]{2}{*}{ FOA1 } & F: GCTTACATCGTTGGAGAAG \\
\hline & R:GCACAGAACTTGGAACATAA \\
\hline \multirow[t]{2}{*}{$A B F 3$} & F: AATGGCGGATTCTATGGATT \\
\hline & R: GCATCTGTAGTGGCTGAG \\
\hline \multirow[t]{2}{*}{ OST1 } & F: GGATCAACCGGGCCAAAG \\
\hline & R: TGAGTGCCTGCAGGAGGAA \\
\hline \multirow[t]{2}{*}{$A B I 1$} & F:AGCTGCTGATATAGTCGTCGTTGATA \\
\hline & R:GAGGATCAAACCGACCATCTAACA \\
\hline \multirow[t]{2}{*}{$R D 22$} & F: GACCATTGAGGAGTGTGAA \\
\hline & R: TCGGTGCGTTCTTCTTAG \\
\hline \multirow[t]{2}{*}{$R D 29 A$} & F: AGGAACCACCACTCAACA \\
\hline & R: GCTCGTCATCATCATCATCT \\
\hline \multirow[t]{2}{*}{$R D 29 B$} & F: AAGGAGACGCAACAAGGG \\
\hline & R: ACGGTGGTGCCAAGTGAT \\
\hline \multirow[t]{2}{*}{ ACTIN2 } & F: CACTGTGCCAATCTACGAGGGT \\
\hline & R: CACAAACGAGGGCTGGAACAAG \\
\hline
\end{tabular}


provide clues to its biological functions. To analyze the expression of FOAl in different organs of Arabidopsis, roots, stems, cauline leaves, flowers and petioles were collected separately from the wild type (Col-0) during the flowering period. Total RNA was isolated and used in real-time fluorescence quantitative PCR analysis. The results showed that FOAl is expressed in all the tested organs, and the relative expression level is higher in roots and flowers and lower in the stem (Figure 1), indicating that FOA1 might play a role in plant root and flower development.

\subsection{Expression of $\mathrm{FOA1}$ is induced by exogenous $\mathrm{ABA}$ and $\mathrm{NaCl}$}

There is an ABRE cis-element in the promoter sequence of FOAl gene, thus the expression of FOAl in response to $\mathrm{ABA}$ and $\mathrm{NaCl}$ was analyzed. Real-time fluorescence quantitative PCR analysis showed that $\mathrm{ABA}$ and $\mathrm{NaCl}$ induced the expression of FOA1 rapidly, peaking at 4 and $2 \mathrm{~h}$, respectively. FOAl expression decreased with the treatment time (Figure 2), suggesting that FOAl might be an ABA signaling-related gene, and is involved in the plant stress response.

\subsection{Molecular identification of a T-DNA insertion mu- tant and $F O A 1$ over-expression lines}

To further study the biological function of FOA1 in ABA

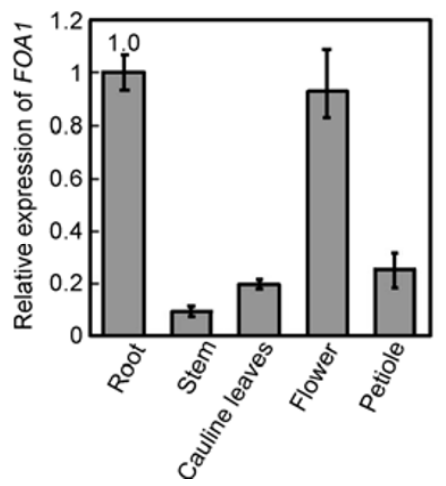

Figure 1 Real-time fluorescence quantitative PCR analysis of FOA1 expression in different organs of Arabidopsis. 1.0 symbolizes that the relative expression level of FOAl in the root is set as 1 .

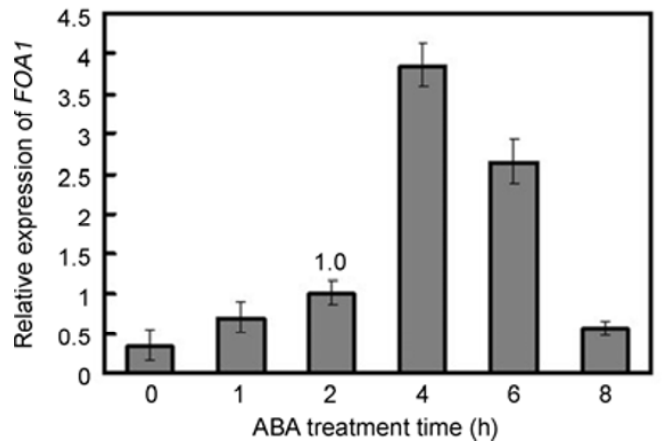

signaling, T-DNA insertion mutant CS857416 was purchased from the ABRC. The sequence of the T-DNA flanking region showed that the insertion was located $599 \mathrm{bp}$ downstream of the ATG start codon (Figure 3A). Homozygous individuals were identified by PCR with primers $\mathrm{R}, \mathrm{F}$ and R0, and four individuals were identified to be homozygote and named foal (Figure 3B). The expression of FOAl in foal mutant was analyzed by semi-quantitative RT-PCR. The result showed that the FOAl signal in the wild type was strong, but the corresponding signal in the foal mutant was barely detectable (Figure $3 \mathrm{C}$ ), indicating that the T-DNA insertion severely impaired FOAl expression.

In addition, the FOA1 coding sequence was subcloned into vector pEarleyGate 203 (N-Myc) downstream of the CaMV35S promoter, and introduced into Arabidopsis mutant $s g s$ (subsequently, sgs refers to the $s g s$ mutant lines expressing wild-type levels of FOAl in this study). Homozygous transgenic lines were identified by Basta resistant selection and real-time fluorescence quantitative PCR analysis. As shown in Figure 3D, FOA1 is overexpressed in FOAlox 1 and FOAlox 2 transgenic lines, and its expression level is about 5 and 25 times higher, respectively, compared with the wild-type sgs.

\subsection{FOA1 is involved in the ABA-mediated inhibition of seed germination and root elongation}

To elucidate the role of FOA1 in ABA signaling, seeds of the wild type, the foal mutant, and over-expression lines FOAlox1 and FOAlox2 were plated on MS solid medium with different concentrations of ABA. The germination rate was scored 5 days later. The foal mutant displayed a reduced germination rate compared with wild-type Col-0 (Figure 4A). Seed germination was seriously inhibited in the presence of increasing concentrations of ABA, and decreased by $25 \%$ and $20 \%$ in foal and Col-0, respectively, and $22 \%, 31 \%$, and $36 \%$ in FOAloxl, FOAlox2, and sgs, respectively, in the presence of $0.6 \mu \mathrm{mol} \mathrm{L}{ }^{-1} \mathrm{ABA}$ (Figure $4 \mathrm{~B}$ ), which indicated that foal was more sensitive to ABA than Col-0, and the FOAloxl and FOAlox 2 were less sensitive to ABA compared with sgs in terms of seed germination.

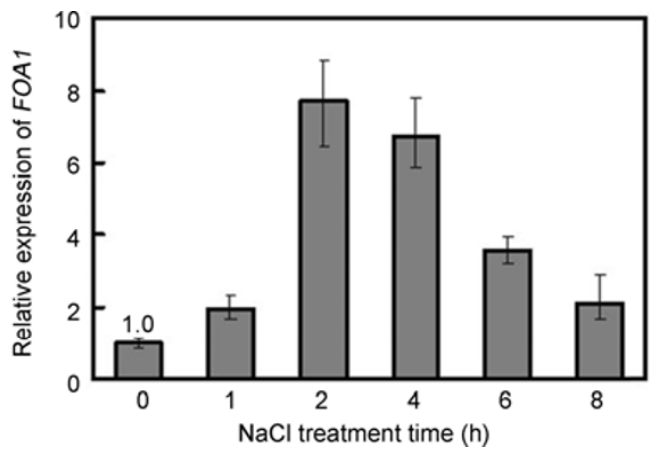

Figure 2 Real-time fluorescence quantitative PCR analysis of the expression of FOA1 in response to exogenous ABA and NaCl. 1.0 symbolizes that the relative expression level of FOA1 in the indicated time point is set as 1. 

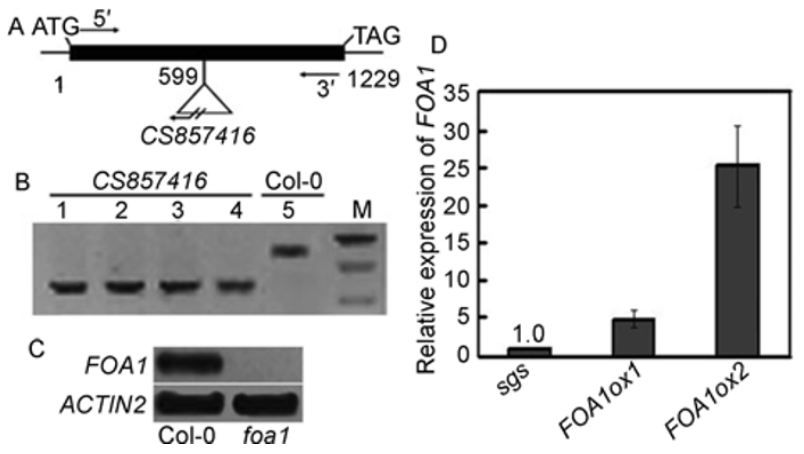

Figure 3 Molecular identifications of T-DNA insertion mutant and FOA1 transgenic plants. A, Schematic of the FOA1 gene and the T-DNA location in the T-DNA insertion mutant CS857416. B, Electrophoresis analysis for identifying homozygous individuals of the T-DNA insertion mutant by PCR. 1-4, 5, and M symbolize homozygous individuals of T-DNA insertion mutant, wild-type Col-0, and DNA marker, respectively. C, RT-PCR analysis of FOA1 expression in foal mutant. ACTIN2 was used as an internal control. D, Real-time fluorescence quantitative PCR analysis of FOA1 expression in FOAloxl and FOA1ox2 transgenic lines. 1.0 symbolizes that the relative expression level of FOAl in wild-type $s g s$ is set as 1 .

Similar to the seed germination assay results, foal showed shorter main roots, but FOAloxl, FOAlox2 showed longer main roots, and both showed an altered response to $\mathrm{ABA}$ in terms of root elongation (Figure 5). The roots of foal were significantly more sensitive to $\mathrm{ABA}$ than wildtype Col- 0 in the presence of $10 \mu \mathrm{mol} \mathrm{L}{ }^{-1} \mathrm{ABA}$, but the roots of FOAloxl and FOAlox 2 showed significantly reduced sensitivity in the presence of 5 and $10 \mu \mathrm{mol} \mathrm{L}^{-1} \mathrm{ABA}$ compared with wild-type sgs (Figure 5). These results indicated that FOA1 might play a negative role in ABA-mediated inhibition of root elongation and seed germination.

\subsection{FOA1 is involved in ABA-mediated control of sto- matal closure and water loss}

ABA is known to induce stomatal closure. To investigate whether FOA1 is involved in ABA-mediated control of stomatal closure, leaves of the wild type, foal, FOAloxl and FOA1ox2 were sampled and immersed in stomatal opening buffer for $10 \mathrm{~h}$ to induce full stomatal opening. They were then treated for another $2 \mathrm{~h}$ in the presence of different concentrations of ABA. The results showed that the stomatal aperture of foal was larger than that of the wild type without ABA treatment $\left(0 \mu \mathrm{mol} \mathrm{L}^{-1} \mathrm{ABA}\right)$ (Figure $\left.6 \mathrm{~A}\right)$. foal exhibited hypersensitivity to $\mathrm{ABA}$, and the ratio of the longitudinal to the traverse diameter of the stomata in foal was lower than that in the wild-type Col-0 in the presence of $10 \mu \mathrm{mol} \mathrm{L}{ }^{-1} \mathrm{ABA}$ (Figure 6B). By contrast, the stomatal
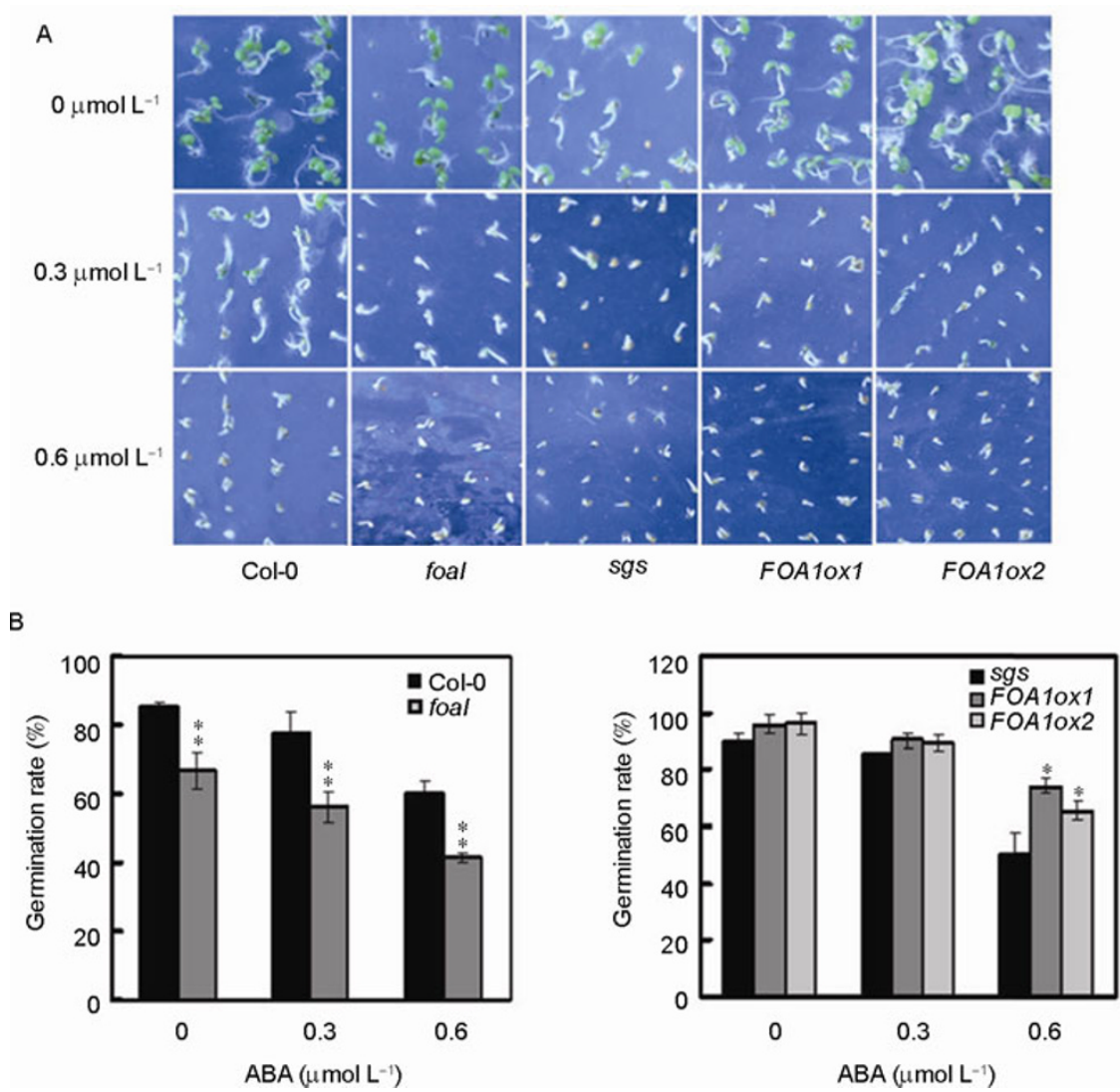

Figure 4 Seed germination of wild type, foal, FOA1oxl and FOA1ox2 in response to ABA. A, Pictures of seed germination of wild type, foal, FOA1ox1 and FOA1ox2 grown on MS solid medium for $5 \mathrm{~d}$. B, Germination rates of wild type, foal, FOA1ox1 and FOAlox2 scored when grown on MS solid medium for $5 \mathrm{~d}$. * and ** indicate that the value of a $t$-test was $P<0.05$ and $P<0.01$ respectively, and indicate a significant difference between the wild type and mutant or overexpression lines. 
apertures of FOA1oxl and FOAlox2 were smaller than wild-type $s g s$ without ABA treatment (Figure 6A). In the presence of $10 \mu \mathrm{mol} \mathrm{L}{ }^{-1} \mathrm{ABA}$, the longitudinal to traverse diameter ratio of stomata in wild-type $s g s$ decreased significantly, but was almost unchanged in FOAloxl and FOA1ox2 (Figure 6B). These results indicated that FOA1 might also play a negative role in ABA-regulated stomatal closure.

Water loss triggered by ABA through stomatal closure is a crucial survival mechanism for plants in response to drought stress [16,27]. To investigate the role of FOAl in regulating water loss, leaves of the wild type, foal, FOA1ox1 and FOA1ox2 were collected and placed in a temperature controlled growth chamber. The weight of the detached leaves was measured at 30 min intervals. Inconsistent with the stomatal aperture results, the water-loss rate of foal was about 1.5 times higher than that of wild-type Col-0. The water-loss rate of FOAloxl and FOAlox2 was about 1.5-2 times lower than that of wild-type sgs (Figure 7 ), which might be due to the larger stomatal aperture of foal compared with wild-type Col-0, and the smaller stomatal aperture of FOAloxl and FOAlox2 compared with wild-type $s g s$ before ABA treatment (Figure 6).

\subsection{FOA1 is involved in ABA's effect on proline accu- mulation in plants}

Exogenous ABA treatment leads to proline accumulation in
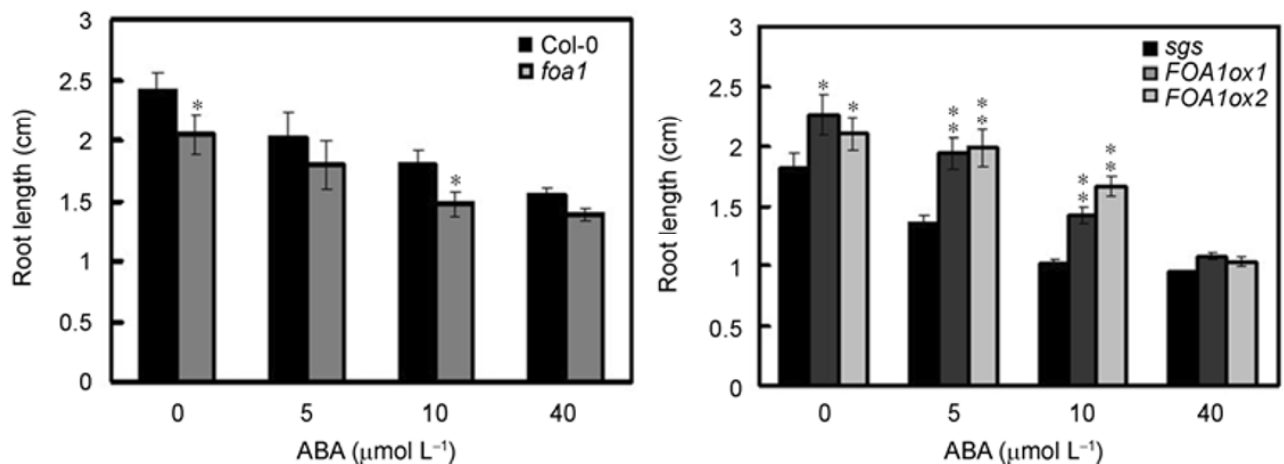

Figure 5 Root lengths of wild type, foal, FOA1oxl and FOA1ox2 in response to ABA. Data represent the mean root length of at least 20 seedlings. * and ** indicate that the value of a $t$-test was $P<0.05$ and $P<0.01$ respectively, and indicate a significant difference between the wild type and the mutant or over expression lines.
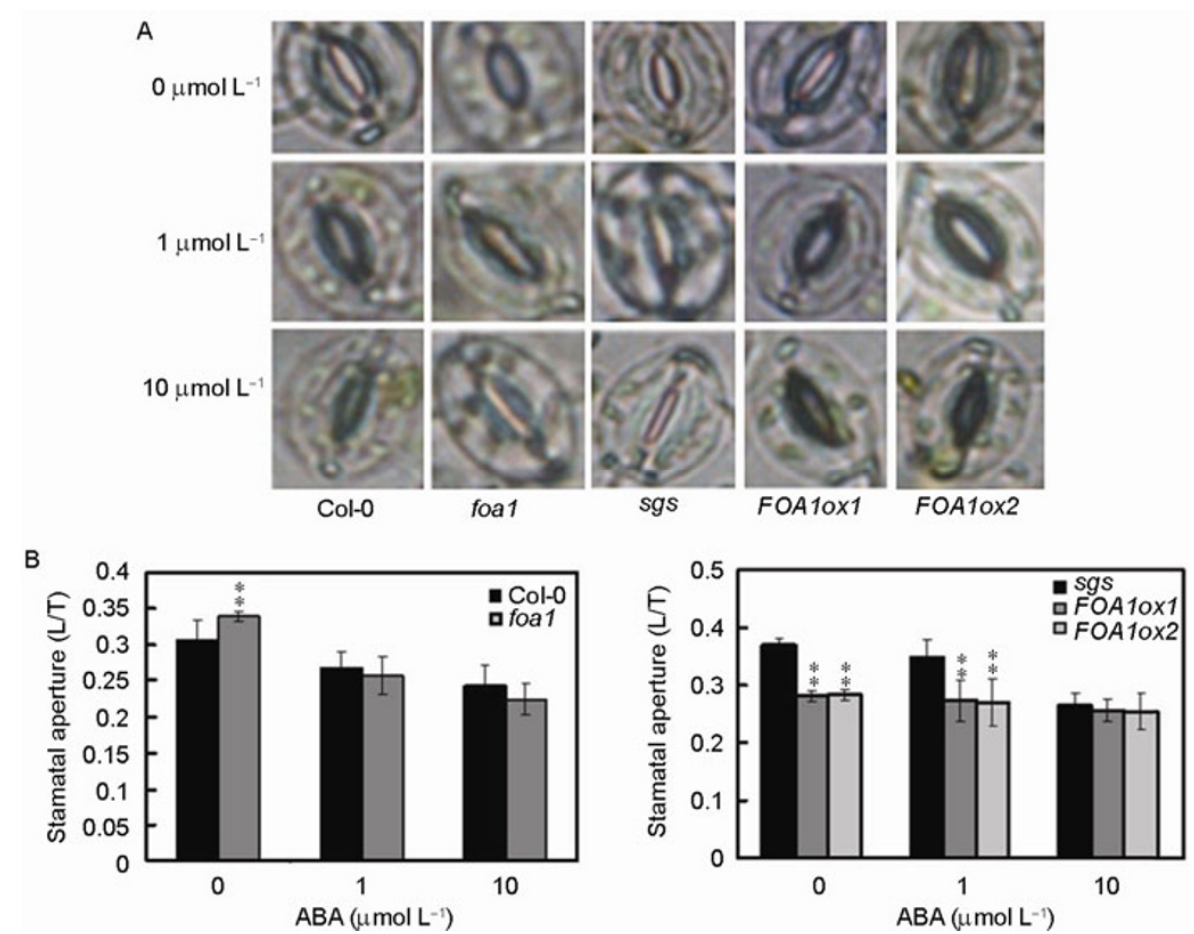

Figure 6 Stomatal aperture of the wild type, foal, FOA1ox1 and FOA1ox2 in response to ABA. A, Pictures of stomata of the wild type, foal, FOA1ox1 and FOAlox 2 in the presence of different concentrations of ABA. B, Data are expressed as the ratio of the longitudinal diameter to the traverse diameter $(\mathrm{L} / \mathrm{T})$ of stomata. * and ** indicate that the value of a $t$-test was $P<0.05$ and $P<0.01$ respectively, and indicate a significant difference between the wild type and the mutant or overexpression lines. 
plants, including barley, maize, rice, soybean and Arabidopsis [28]. To investigate whether FOA1 affects proline accumulation in plants in response to $\mathrm{ABA}$, the proline contents of the wild type, foal, FOAloxl, and FOAlox 2 plants in response to ABA were determined. As shown in Figure 8 , proline accumulated in plants in the presence of ABA, but there were significant differences between the wild type and the mutants. Proline content increased by 1.2 and 1 times in foal mutant and wild-type Col- 0 respectively, and increased by 0.7 and 1.6 times in FOAl overexpression lines and wild-type sgs respectively (Figure 8). These results indicated that foal accumulated significantly more proline than wild-type Col-0, while FOAloxl and FOA1ox2 accumulated significantly less proline than wild-type $s g s$. Thus, FOA1 might also play a negative regulatory role in ABA's control of proline accumulation.

\subsection{FOA1 regulates the expression of $\mathrm{ABA}$ and stress- responsive genes}

To provide molecular evidence of FOA1's involvement in ABA signal transduction, the expressions of a series of ABA-, drought-, and salt stress-related genes were analyzed in the wild type and foal mutant treated with or without ABA. The genes included transcription factors $A B I 1$ [29] and $A B F 3$ [30], $A B F 3$ interaction protein kinase OST1 [31], and ABA and stress-responsive genes $R D 29 A, R D 29 B$ and $R D 22[32,55]$. The results showed that the expressions of all tested genes were induced by ABA, but there were differences between foal and the wild-type Col-0 (Figure 9). The expression levels of $A B I 1, O S T 1$ and $A B F 3$ in foal were higher, and the expression levels of $R D 29 A, R D 29 B$ and $R D 22$ in foal were lower than that in the wild-type Col-0 (Figure 9). These results indicated that a null mutation of FOAl could result in altered expression of ABA, drought, salt stress-responsive genes, which further supported the hypothesis that FOAl is an ABA signaling-related gene, which might play a role in stress resistance of plants through the ABA signaling pathways.

\section{Discussion}

As members of the SCF complex, F-box proteins control many hormone signal transductions, including GA [7], auxin $[9,10]$, and ethylene signal transduction by identifying target proteins and mediating their degradation. Recently, certain F-box proteins were also found to be involved in ABA signal transduction [34]. F-box protein EDL3 functions as a positive regulator in ABA signal transduction and regulates seed germination, root growth, etiolated seedlings turning green, and flowering [8]. TLP9 may be involved in
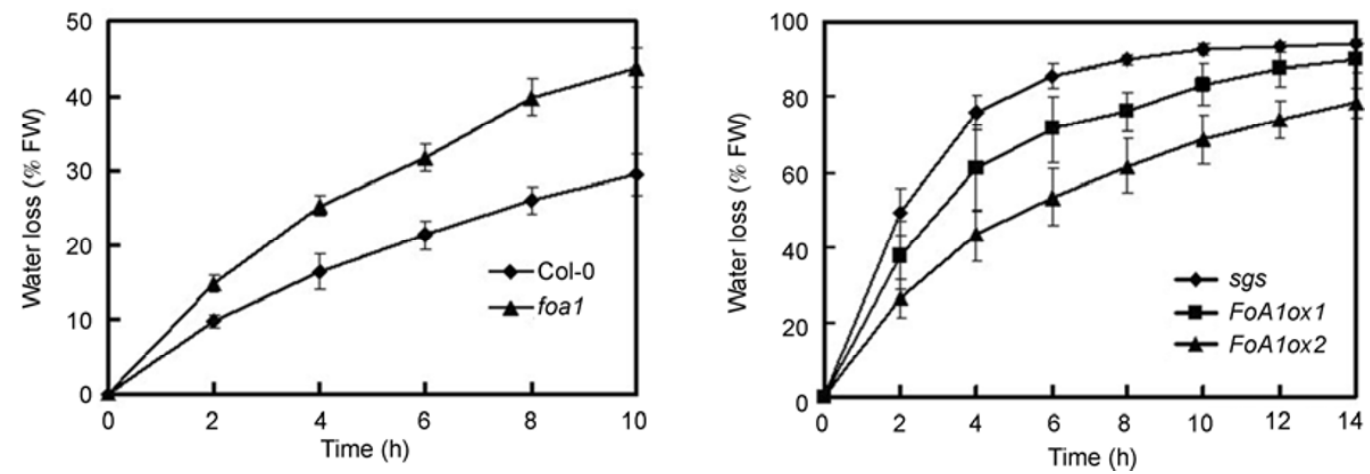

Figure 7 Water loss of the wild type, foal, FOA1ox1 and FOA1ox2 in response to ABA. Water loss is expressed as a percentage of the initial fresh weight at indicated intervals. FW, fresh weight.
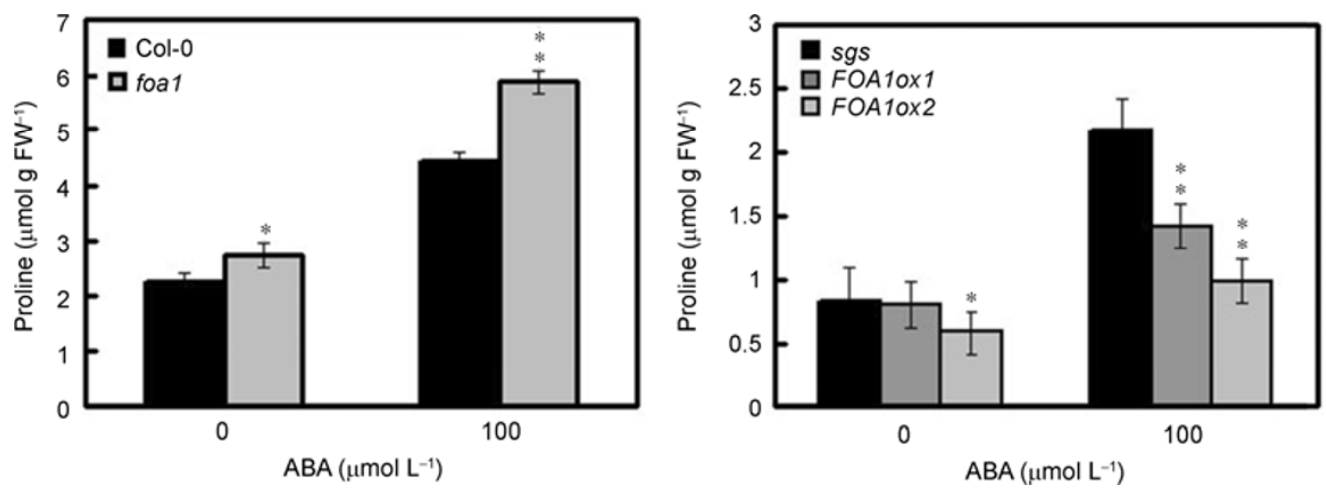

Figure 8 Proline accumulation of wild type, foal, FOAloxl and FOA1ox2 in response to ABA. FW, fresh weight. * and ** indicate that the value of a T test was $P<0.05$, and $P<0.01$ respectively, and indicate a significant difference between the wild type and the mutant or overexpression lines. 

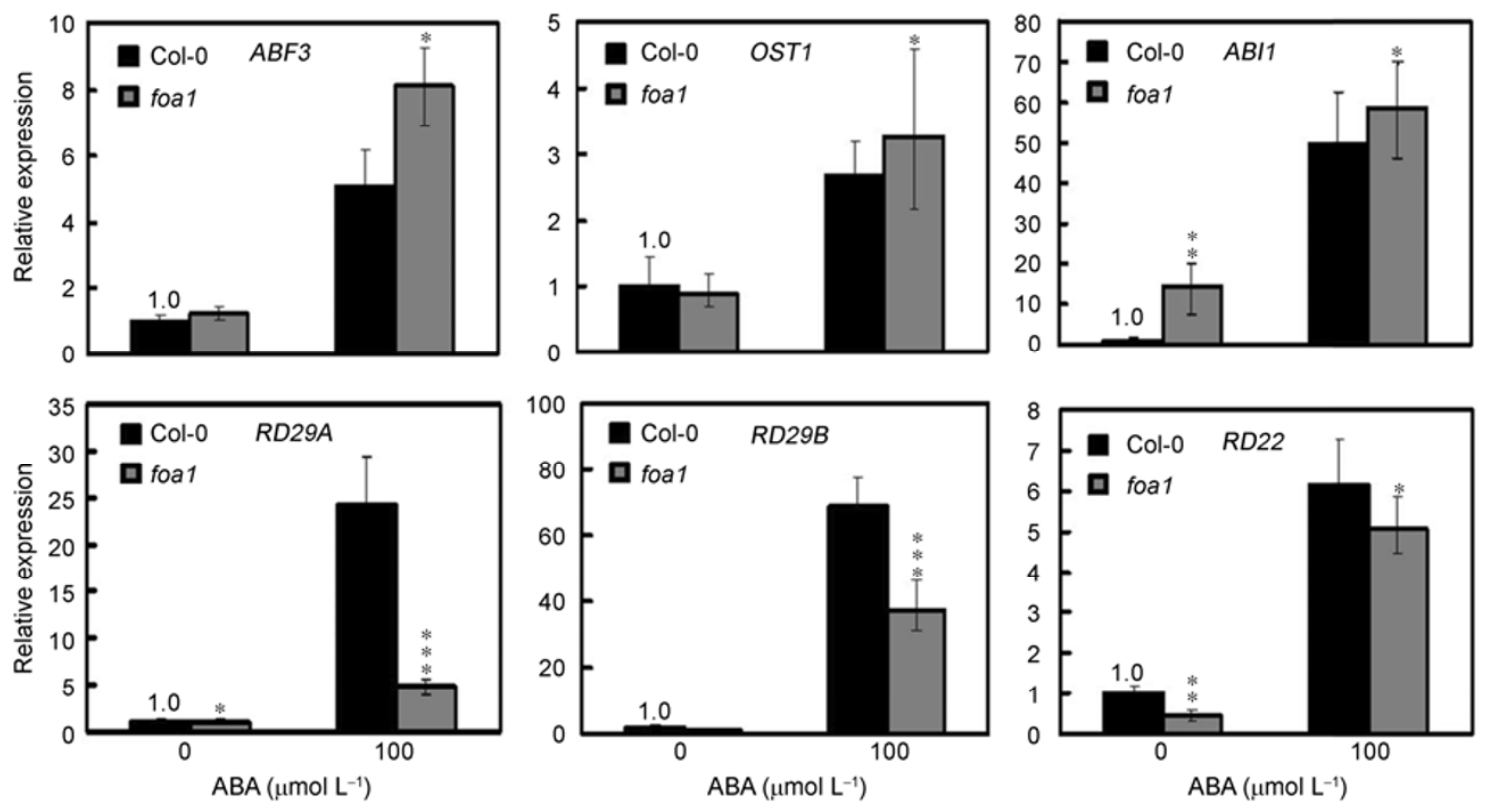

Figure 9 Expressions of ABA and stress-responsive genes in wild-type Col-0 and foal mutant in response to ABA. 14-day-old wild-type and foal seedlings were treated with $100 \mu \mathrm{mol} \mathrm{L} \mathrm{L}^{-1} \mathrm{ABA}$ for 0 or $6 \mathrm{~h}$, and sampled for RNA isolation. The expressions of ABA and stress responsive genes were analyzed by real-time fluorescence quantitative PCR. *, **, and *** indicate that the value of a $t$-test was $P<0.05, P<0.01$ and $P<0.001$, respectively, and indicate significant differences between the wild type and the mutant. 1.0 symbolizes that the relative expression level of FOA1 in Col-0 without ABA treatment is set as 1 .

ABA-mediated reactions by identifying negative regulators of ABA signal transduction. The TLP9 mutant showed loss of sensitivity to ABA and TLP9 over-expression lines showed hypersensitivity to ABA [35]. A null mutation of DOR leads to stomatal closure and enhanced droughtresistance. By comparison, overexpression of DOR in Arabidopsis resulted in hypersensitivity to drought stress. These results indicated that DOR plays a negative role in response to drought stress through ABA signal transduction [16]. In this study, we reported that FOAl is an ABA signaling-related gene, involved in many ABA-regulated Arabidopsis biological processes, including seed germination, root elongation, stomatal closure, water-loss rate and proline accumulation.

The expression pattern of the F-box gene in different organs was analyzed. FOAl is expressed in all tested organs, and showed the highest transcript level in roots and flowers (Figure 1). Phenotype analysis showed that the FOAI null mutant foal has a shorter root phenotype, while the FOAl over-expression lines FOAloxl and FOAlox 2 exhibited a longer root phenotype (Figure 5), which is consistent with the organ expression result, and indicated that FOAl might be involved in plant root development. No flower phenotype was found in the foal mutant or in the FOAloxl and FOA1ox 2 overexpression lines in this study.

There is an ABRE element in the promoter region of $F O A 1$, and the expression analysis results proved that exogenous $\mathrm{ABA}$ and $\mathrm{NaCl}$ rapidly induced the transcription of FOA1, which indicated that FOAl is probably an ABA signaling-related gene. Phenotype analysis showed that the
FOAI null mutant foal exhibited increased sensitivity and FOAloxl and FOAlox2 overexpression lines exhibited decreased sensitivity to ABA in the seed germination, root elongation, stomatal closure assays compared with the wild type. foal accumulated significantly more proline than wild type Col-0, while FOAloxl and FOA1ox2 accumulated significantly less proline than the wild-type $s g s$ in the presence of ABA. These results suggested that FOAl plays a negative role in ABA signal transduction. The proline content of plants is a characteristic feature of stress resistance [28]. Thus, our results suggested that FOA1 is involved in ABA-dependent stress resistance. Differences between Col-0 and sgs in response to ABA were also discovered in this study (Figures 4-6), which may be a result of the recessive monogenic mutation carried by the sgs mutant [23].

The expression analysis of stress-responsive genes showed that $A B F 3$, which is a positive regulatory transcription factor in ABA signal pathway [30,36], is expressed higher in the foal mutant than in the wild-type Col-0. OST1, which activates ABF3 and is activated by ABA [32], is also expressed higher in the foal mutant than in the wild type, suggesting that ABA signal transduction is enhanced in the foal null mutant, which was consistent with the phenotype of foal (hypersensitivity to ABA). ABIl is a group A PP2C in Arabidopsis and has been described as a negative regulator of ABA signaling. $A B I I$ is strongly induced by ABA $[8,29,36,37]$; its transcription level increased in lines overexpressing $A B F 3, A B F 4$ [30], or TSK1 [36] in which ABA signaling is enhanced, while it decreased in ABA-insensi- 
tive mutants such as abi5-1 and snrk2.2/snrk2.3/snrk2.6 triple mutant, in which ABA signaling is blocked [38]. Consistent with previous results, the expression of $A B I 1$ increased in the ABA hypersensitive mutant foal. The expressions of $R D 29 A, R D 29 B$ and $R D 22$ are strongly induced by salt, drought, cold and ABA [32,33]. Similar results were obtained in this study; the transcript levels of RD29A, $R D 29 B$, and $R D 22$ increased in both the wild type and foal in response to ABA. However, their expression levels in foal were lower than in the wild-type Col-0. These results suggested that a null mutation of FOAl resulted in altered transcript levels of $\mathrm{ABA}$ and stress-responsive genes, providing further evidence that FOAl is an ABA signaling-related gene, and might be involved in the plant stress response.

The FOA1 protein carries an F-box domain and is thus presumed to act as a component of the SCF complex. We believe it is involved in ABA signal transduction by targeting transcription factors that regulate the expression of ABA and stress-responsive genes for degradation in the proteasome. This hypothesis requires to be further tested.

We thank Professor Lin ChenTao for his kind guidance of this work. This work was supported by the National Natural Science Foundation of China (Grant No. 31171176), Research Fund for the Doctoral Program of Higher Education of China (Grant No. 755228001), Natural Science Foundation of Hunan Province (Grant No. 11JJA002), and the National Key Laboratory of Plant Molecular Genetics (Grant No. Y109Z711T1).

1 Gagne J M, Downes B P, Shiu S, et al. The F-box subunit of the SCF E3 complex is encoded by a diverse superfamily of genes in Arabidopsis. Proc Natl Acad Sci USA, 2002, 99: 11519-11524

2 Lechner E, Achard P, Vansiri A, et al. F-box proteins everywhere. Curr Opin Plant Biol, 2006, 9: 631-638

3 Smalle J, Vierstra R D. The ubiquitin $26 \mathrm{~S}$ proteasome proteolytic pathway. Annu Rev Plant Biol, 2004, 55: 555-590

4 Somers D E, Fujiwara S. Thinking outside the F-box: novel ligands for novel receptors. Trends Plant Sci, 2009, 14: 206-213

5 Petroski M D, Deshaies R J. Function and regulation of cullin-RING ubiquitin ligases. Nat Rev Mol Cell Biol, 2005, 6: 9-20

6 Schwechheimer C, Willige B C, Zourelidou M, et al. Examining protein stability and its relevance for plant growth and development. Methods Mol Biol, 2009, 479: 147-171

7 Wang F, Deng X W. Plant ubiquitin-proteasome pathway and its role in gibberellin signaling. Cell Res, 2011, 21: 1286-1294

8 Koops P, Pelser S, Ignatz M, et al. EDL3 is an F-box protein involved in the regulation of abscisic acid signalling in Arabidopsis thaliana. J Exp Bot, 2011, 62: 5547-5560

9 Potuschak T, Lechner E, Parmentier Y, et al. EIN3-dependent regulation of plant ethylene hormone signaling by two Arabidopsis F-box proteins: EBF1 and EBF2. Cell, 2003, 115: 679-689

10 Kepinski S, Leyser O. The Arabidopsis F-box protein TIR1 is an auxin receptor. Nature, 2005, 435: 446-451

11 Samach A, Klenz J E, Kohalmi S E, et al. The UNUSUAL FLORAL ORGANS gene of Arabidopsis thaliana is an F-box protein required for normal patterning and growth in the floral meristem. Plant J, 1999, 20: 433-445

12 Ni W, Xie D, Hobbie L, et al. Regulation of flower development in Arabidopsis by SCF complexes. Plant Physiol, 2004, 134: 1574-1585

13 Lee J H, Kim W T. Regulation of abiotic stress signal transduction by E3 ubiquitin ligases in Arabidopsis. Mol Cells, 2011, 31: 201-208

14 Lyzenga W J, Stone S L. Abiotic stress tolerance mediated by protein ubiquitination. J Exp Bot, 2011, 63: 599-616

15 Yan Y S, Chen X Y, Yang K, et al. Overexpression of an F-box protein gene reduces abiotic stress tolerance and promotes root growth in rice. Mol Plant, 2011, 4: 190-197

16 Zhang Y, Xu W Y, Li Z H, et al. F-box protein DOR functions as a novel inhibitory factor for abscisic acid-induced stomatal closure under drought stress in Arabidopsis. Plant Physiol, 2008, 148: 2121-2133

17 Sharma N, Shen H, Luong P, et al. Regulation of drought tolerance by the F-box protein MAX2 in Arabidopsis. In: Joint Annual Meeting of the American Society of Plant Biologists, Meridia, Mexico, 2008. 126

18 Jia Y, Gu H, Wang X, et al. Molecular cloning and characterization of an F-box family gene CarF-box 1 from chickpea (Cicer arietinum L.). Mol Biol Rep, 2011, 39: 2337-2345

19 Ning Y, Wang G, Xie Q. E3 ubiquitin ligase-mediated drought responses in plants (in Chinese). Chin Bull Bot, 2011, 46: 606616

20 Calderon-Villalobos L I, Nill C, Marrocco K, et al. The evolutionarily conserved Arabidopsis thaliana F-box protein AtFBP7 is required for efficient translation during temperature stress. Gene, 2007, 392: 106-116

21 Kuroda H, Takahashi N, Shimada H, et al. Classification and expression analysis of Arabidopsis F-Box-containing protein genes. Plant Cell Physiol, 2002, 43: 1073-1085

22 Clough S J, Bent A F. Floral dip: a simplified method for Agrobacterium-mediated transformation Arabidopsis thaliana. Plant J, 1998, 16: 735-743

23 Elmayan T, Balzergue S, Beon F, et al. Arabidopsis mutants impaired in cosuppression. Plant Cell, 1998, 10: 1747-1758

24 Pei Z M, Kuchitsu K, Ward J M, et al. Differential abscisic acid regulation of guard cell slow anion channels in Arabidopsis wild-type and abi1 and abi2 mutants. Plant Cell, 1997, 9: 409-423

25 Shan H, Chen S, Jiang J, et al. Heterologous expression of the chrysanthemum R2R3-MYB transcription factor CmMYB2 enhances drought and salinity tolerance, increases hypersensitivity to ABA and delays flowering in Arabidopsis thaliana. Mol Biotechnol, 2011, doi: 10.1007/s12033-011-9451-1

26 Serpil Ü, Yúksel K, Elif Ü. Proline and ABA levels in two sunflower genotypes subjected to water stress. Bulg J Plant Physiol, 2004, 30: 34-47

2736 Kim Y Y, Jung K W, Yoo K S, et al. A stress-responsive caleosin-like protein, AtCLO4, acts as a negative regulator of ABA responses in Arabidopsis. Plant Cell Physiol, 2011, 52: 874-884

28 Savouré A, Hua X J, Bertauche N, et al. Abscisic acid-independent and abscisic acid-dependent regulation of proline biosynthesis following cold and osmotic stresses in Arabidopsis thaliana. Mol General Genet, 1997, 254: 104-109

29 Leung J, Merlot S, Giraudat J. The Arabidopsis ABSCISIC ACID-INSENSITIVE2 (ABI2) and ABI1 genes encode homologous protein phosphatases $2 \mathrm{C}$ involved in abscisic acid signal transduction. Plant Cell, 1997, 9: 759-771

30 Kang J Y, Choi H I, Im M Y, et al. Arabidopsis basic leucine zipper proteins that mediate stress responsive abscisic acid signaling. Plant Cell, 2002, 14: 343-357

31 Sirichandra C, avanture M, Turk B E, et al. The Arabidopsis ABA-activated kinase OST1 phosphorylates the bZIP transcription factor $\mathrm{ABF} 3$ and creates a 14-3-3 binding site involved in its turnover. PLoS ONE, 2010, 5: e13935

32 Yamaguchi-Shinozaki K, Shinozaki K. A novel cis acting element in an Arabidopsis gene is involved in responsiveness to drought, lowtemperature, or high-salt stress. Plant Cell, 1994, 6: 251-264

33 Yamaguchi-Shinozaki K, Shinozaki K. The plant hormone abscisic acid mediates the drought-induced expression but not the seedspecific expression of $\mathrm{rd} 22$, a gene responsive to dehydration stress in Arabidopsis thaliana. MOI Gen Genet, 1993, 238: 97-105

34 Liu H, Stone S L. E3 ubiquitin ligases and abscisic acid signaling. Plant Signal Behav, 2011, 6: 344-348

35 Lai C P, Lee C L, Chen P H, et al. Molecular analyses of the Ara- 
bidopsis TUBBY-like protein gene family. Plant Physiol, 2004, 134:1586-97

36 Li C, Liu Z, Zhang Q, et al. SKP1 is involved in abscisic acid signalling to regulate seed germination, stomatal opening and root growth in Arabidopsis thaliana. Plant Cell Environ, 2011, doi: 10.1111 /j.1365-3040.2011.02464.x

37 Yang X. Heterologous expression of the chrysanthemum R2R3-MYB transcription factor CmMYB2 enhances drought and salinity toler- ance, increases hypersensitivity to ABA and delays flowering in Arabidopsis thaliana. Mol Biotechnol, 2011, doi: 10.1007/s12033011-9451-1

38 Nakashima K, Fujita Y, Kanamori N, et al. Three Arabidopsis SnRK2 protein kinases, SRK2D/SnRK2.2, SRK2E/ SnRK2.6/OST1 and SRK2I/SnRK2.3, involved in ABA signaling are essential for the control of seed development and dormancy. Plant Cell Physiol, 2009, 50: $1345-1363$

Open Access This article is distributed under the terms of the Creative Commons Attribution License which permits any use, distribution, and reproduction in any medium, provided the original author(s) and source are credited. 